\title{
Comparison of anticandidal activity of Punica granatum (Pomegranate) and Lawsonia inermis (Henna leaves): An in-vitro study
}

\author{
Singla $S^{1 *}$, Gupta $R^{1}$, Puri $A^{1}$, Singh $V^{2}$, Roy $S^{1}$ \\ ${ }^{I}$ Department of Oral Pathology and Microbiology, Himachal institute of dental sciences; Paonta Sahib (Himachal Pradesh) \\ ${ }^{2}$ Department of Microbiology, Himachal institute of life sciences; Paonta Sahib (Himachal Pradesh) \\ *Corresponding author E-mail: swatisingla2201@gmail.com
}

\begin{abstract}
Background: Oral Candidosis is common opportunistic infection of the oral cavity caused by overgrowth of Candida species, the commonest being Candida albicans. Increasing resistance and side effects of the antifungal drugs used in therapy of candidosis have given way to the use of natural products. Pomegranate and Henna have been found to be very effective in the treatment of Candidosis.

Aim of study: This study evaluates the antifungal activities of pomegranate peel extract (rind), pomegranate seed extract (Punica granatum) and Henna leaves (Lawsonia inermis) against different strains of Candida albicans.

Materials and methods: Both aqueous and ethanolic extracts of pomegranate peel, seeds and henna leaves were prepared. Anticandidal activity was determined and minimum zones of inhibitions (in $\mathrm{mm}$ ) shown by extracts of all the three plants were measured and compared.

Results: Henna leaves showed the maximum zone of inhibition (mean $20 \mathrm{~mm}$ ) followed by pomegranate peels (mean $13.3 \mathrm{~mm}$ ) and seeds (mean $3.75 \mathrm{~mm}$ ). Also, aqueous extracts of henna and pomegranate showed higher antifungal activity as compared to their corresponding ethanolic extracts.

Conclusion: Use of medicinal plants against candida can be a viable alternative to other antifungal agents as they offer a cheap and effective module used in control of oral infections caused by Candida species.
\end{abstract}

\section{Introduction}

Oral Candidosis is an opportunistic infection of the oral cavity. It is common and underdiagnosed among the elderly, particularly in those who wear dentures and in many cases is avoidable with a good mouth care regimen. It can also be a mark of systemic disease, such as diabetes mellitus and is a common problem among the immunocompromised [1].

Oral Candidosis is caused by overgrowth or infection by a yeast-like fungus, candida [2,3]. The important ones are $\mathrm{C}$ albicans, C tropicalis, C glabrata, C pseudotropicalis, C guillierimondii, C krusei, C lusitaniae, C parapsilosis, and C stellatoidea. C albicans, C glabrata, and C tropicalis represent more than $80 \%$ of isolates from clinical infection. Candida albicans is the commonest of all these [1].

Oral Candidosis is the most common human fungal infection especially in early and later life [4,5]. Candida albicans is a normal commensal of the mouth and generally causes no problems in healthy people [1]. Normally present as a yeast, the organism, under favourable conditions like broad-spectrum antibiotic therapy, xerostomia, immune dysfunction (secondary to systemic diseases such as diabetes or the use of immune suppressant medications), or the presence of removable prostheses; has the ability to transform into a pathogenic hyphae form. Furthermore, about one in four patients with lichen planus will have superimposed Candidosis [6].

Candidal overgrowth can lead to local discomfort, altered taste sensation, dysphagia from oesophageal overgrowth (resulting in poor nutrition and prolonged hospital stay). In immunocompromised patients, infection can spread through the bloodstream or upper gastrointestinal tract leading to severe infection with significant morbidity and mortality [1].

Amphotericin B, Nystatin, Fluoconazole, Itraconazole and Clotrimazole are the antifungal drugs commonly and widely used in the treatment of Candidosis [1]. They usually inhibit membrane component sterol biosynthesis (azoles, allylamines and morpholines), directly interact with the cell membrane (polyenes) or target cell wall biosynthesis (echinocandins) [7]. The majority of clinically used antifungals have various drawbacks in terms of toxicity, efficacy 
and cost, and their frequent use has led to the emergence of resistant strain [8]. This has given way to the use of plant products in the treatment of candidosis. Natural products, either as pure compounds or as standardised plant extracts, provide unlimited opportunities for new drug leads because of the matchedless availability of chemical diversity. ${ }^{8}$ Among the natural products, Pomegranate and Henna have been found to be very effective in the treatment of Candidiasis $[9,10]$.

The present study evaluates and compares the antifungal activities of Pomegranate peel extract (rind), Pomegranate seed extract and Henna (Lawsonia inermis) leaves against different strains of Candida albicans.

\section{Materials and methods}

\subsection{Method [11]}

The plants were selected on the basis of their ethnobotanical use and their importance in day-to-day life. The plants/plant parts collected were free from any visible contamination and were healthy and fresh.

\subsubsection{Collection of plant material}

The plant material were collected from Herbal garden HIP, Paonta Sahib. They were thoroughly washed and shade dried at room temperature $\left(37^{\circ} \mathrm{C}\right)$ for about a week. Then, they were grinded to powder (Fig.1a). The dried powder was stored in airtight bottles at $28^{\circ} \mathrm{C}$ for further extraction. $100 \mathrm{gms}$ of the dried plant material were used for the extraction.

\subsubsection{Preparation of ethanolic plant extract}

Coarsely powdered material was weighed and extracted with ethanol using a Soxhlet Apparatus for 5 to 6 hours. A thimble was prepared by using a $0.5 \mathrm{~mm}$ of Whatmann filter paper. $70 \mathrm{~g}$ of dried material was kept in the thimble for extraction. A clean dry pre-weighed round bottom flask was used for the extraction for dry plant material. $150 \mathrm{ml}$ of solvent was used for the process of extraction. The extraction was carried out at the boiling point of the solvent for duration of 12-14 hours (Fig. 1b).

\subsubsection{Preparation of aqueous plant extract}

20 gram of dried ground samples were mixed with $200 \mathrm{ml}$ of water with a magnetic stirrer for 15 min. The extracts were filtered over filter paper. Heated over water bath for 5-6 hours till they become viscous (Fig. 1b).

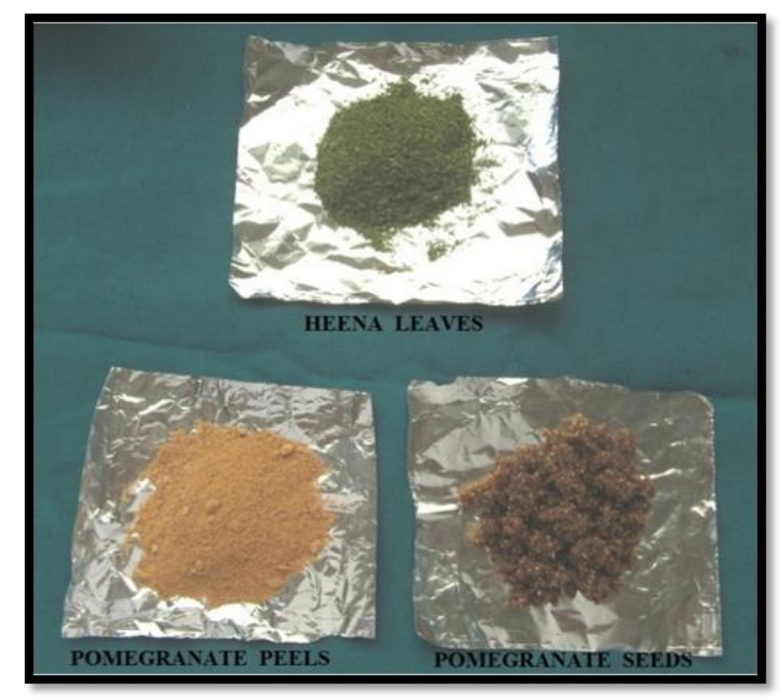

Fig.1a: Plant powders of henna leaves, pomegranate peels and pomegranate seeds

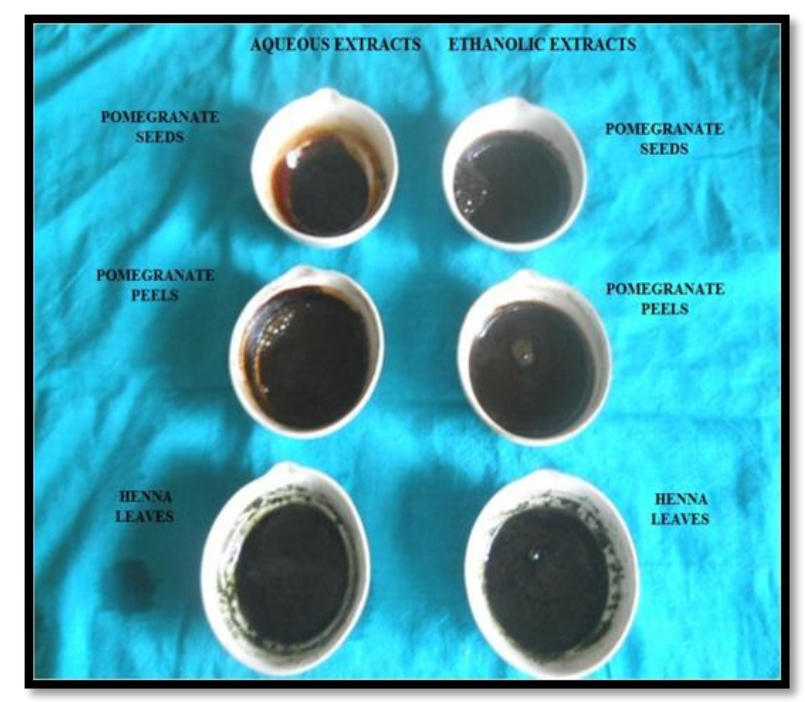

Fig. 1b: Aqueous and ethanolic extracts of henna leaves, pomegranate peels and pomegranate seeds.

\subsubsection{Microorganisms}

Fungal strains were obtained from Microbiological Type Culture Collection and Gene Bank (MTCC, Chandigarh, India). Four fungal strains were used in this study (MTCC 183, MTCC 1637, MTCC 3018, and MTCC 3958) (Fig. 2). They were inoculated in Soybean Casein Digest Medium (SCDM) broth and kept for incubation at $27^{\circ} \mathrm{C}$ for $48 \mathrm{hrs}$. 


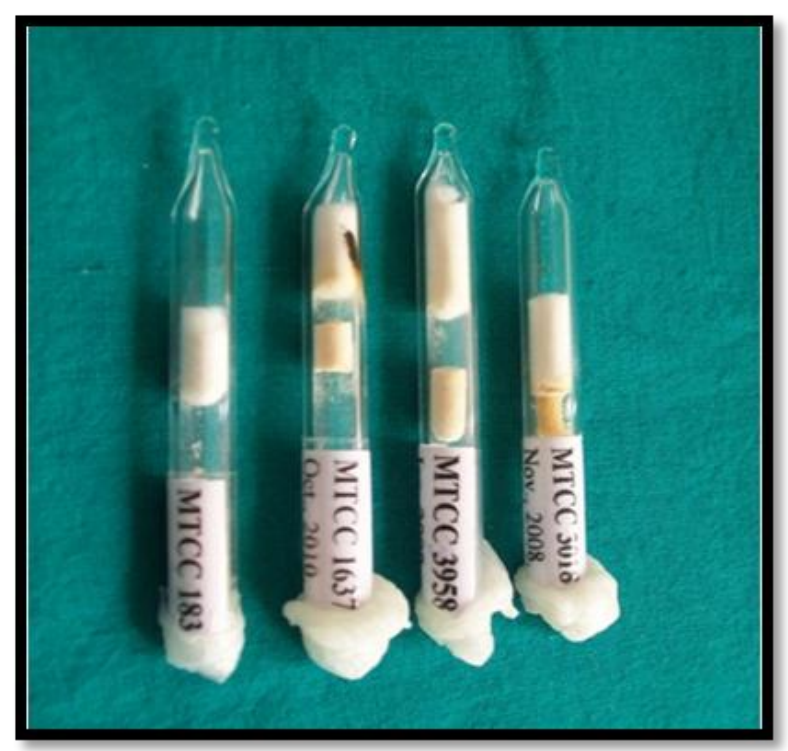

Fig. 2: Candida albicans fungal strains used in the study.

\subsubsection{Agar well diffusion assay}

Potato Dextrose Agar (PDA) was dissolved in distilled water and the medium was sterilized. It was transferred into sterile 6 inch diameter petridishes. Then it was allowed to set at room temperature for about 10 minutes and then kept in incubator at $27^{\circ} \mathrm{C}$ for $48 \mathrm{hrs}$. After incubation, $100 \mu \mathrm{l}$ of fungal strains were uniformly spreaded on PDA plates with the help of sterile spreader. After setting two uniform wells ( $8 \mathrm{~mm}$ diameter each) at a distance of $7 \mathrm{~mm}$ were made in each Petridish. The wells were filled with $200 \mu \mathrm{l}$ of the different extracts. Clotrimazole disc $(20 \mu \mathrm{g})$ was also applied as control along with the plants extracts. The plates thus prepared were kept in an incubator at $27^{\circ} \mathrm{C}$ to allow for the diffusion of the plant extracts. After 48 hours, the agar plates were examined for inhibition zones. The zones were measured in millimeters and the results were tabulated.

\section{Results}

Minimum zones of inhibition were measured for ethanolic and aqueous plant extracts each for henna leaves, pomegranate peels and pomegranate seeds against all the four strains of candida albicans (Fig. 3a). Henna leaves showed the maximum zones of inhibition (mean $20 \mathrm{~mm}$ ) followed by pomegranate peels (mean $13.3 \mathrm{~mm}$ ) and seeds (mean $3.75 \mathrm{~mm}$ ) (Fig. 3b). Out of the aqueous and ethanolic extract, aqueous extract showed higher antifungal activity as compared to that of ethanolic extract (Table 1).

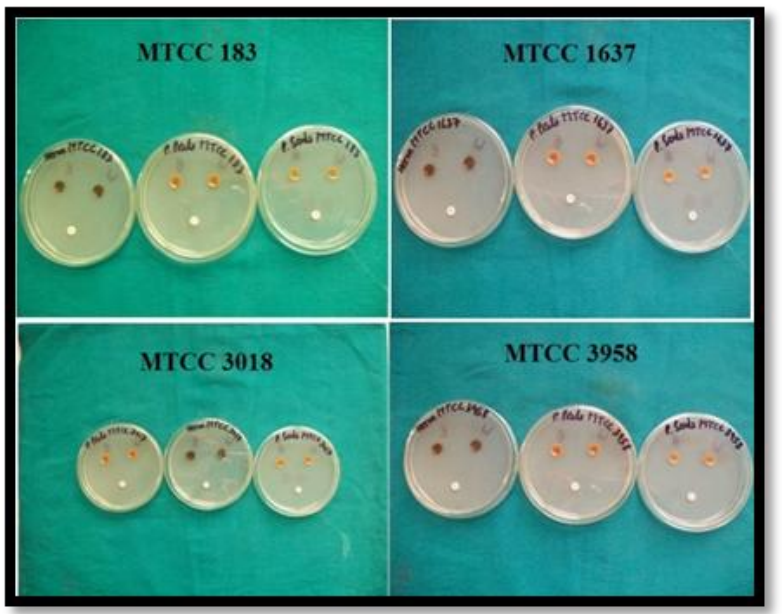

Fig. 3a: Activity (measured as zones of inhibition) of the plant extracts at Zero hours

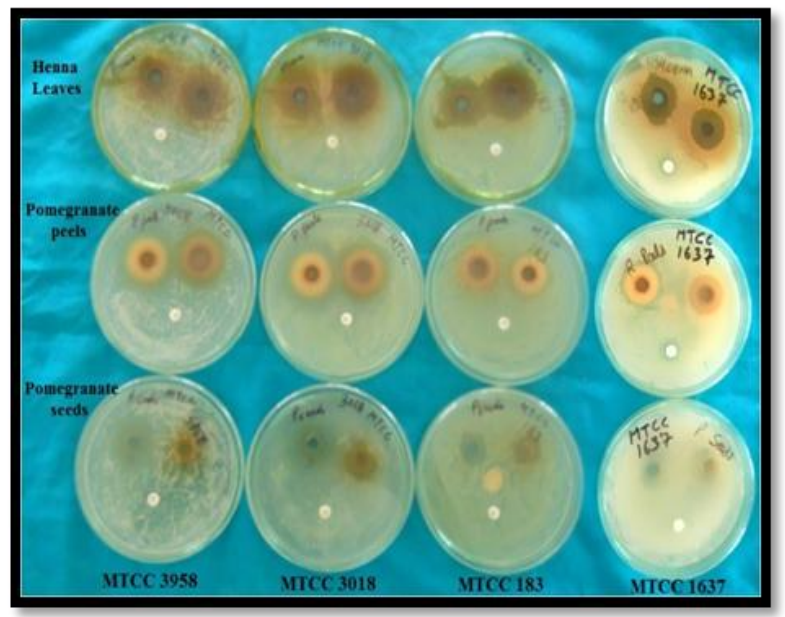

Fig. 3b: Activity (measured as zones of inhibition) of the plant extracts at 48 hours. 


\begin{tabular}{|c|c|c|c|c|c|c|}
\hline \multirow[t]{3}{*}{ Strain no. } & \multicolumn{6}{|c|}{ MINIMUM ZONE OF INHIBITION (in mm) } \\
\hline & \multicolumn{2}{|c|}{ Henna Leaves } & \multicolumn{2}{|c|}{ Pomegranate peels } & \multicolumn{2}{|c|}{ Pomegranate seeds } \\
\hline & $\begin{array}{c}\text { Aqueous } \\
\text { extract }\end{array}$ & $\begin{array}{c}\text { Ethanolic } \\
\text { extract }\end{array}$ & $\begin{array}{c}\text { Aqueous } \\
\text { extract }\end{array}$ & $\begin{array}{c}\text { Ethanolic } \\
\text { extract }\end{array}$ & $\begin{array}{c}\text { Aqueous } \\
\text { extract }\end{array}$ & $\begin{array}{c}\text { Ethanolic } \\
\text { extract }\end{array}$ \\
\hline MTCC 3958 & 23 & 13 & 14 & 11 & 3 & 3 \\
\hline MTCC 3018 & 25 & 16 & 19 & 13 & 4 & 2 \\
\hline MTCC 183 & 26 & 18 & 17 & 10 & 7 & 4 \\
\hline MTCC 1637 & 25 & 14 & 13 & 10 & 3 & 4 \\
\hline
\end{tabular}

\section{Discussion}

Candida, although being a normal oral commensal, may prove to be quite notorious under favourable conditions leading to discomfort in dental patient. In fact, C. albicans may be a component of normal oral microflora, with as many as $30 \%$ to $50 \%$ of people simply carrying the organism in their mouth without any clinical evidence of infection [12].

In the present scenario, incidence of candidosis is increasing day by day due to the increasing resistance against various antifungal drugs used in its treatment. Resistance to azole antifungals was reported in the late 1980s after prolonged therapy with miconazole and ketoconazole [7]. In spite of its widespread use in the medical community, many reports described the clinical failure of Fluconazole therapy in individuals with HIV infection [13,14].

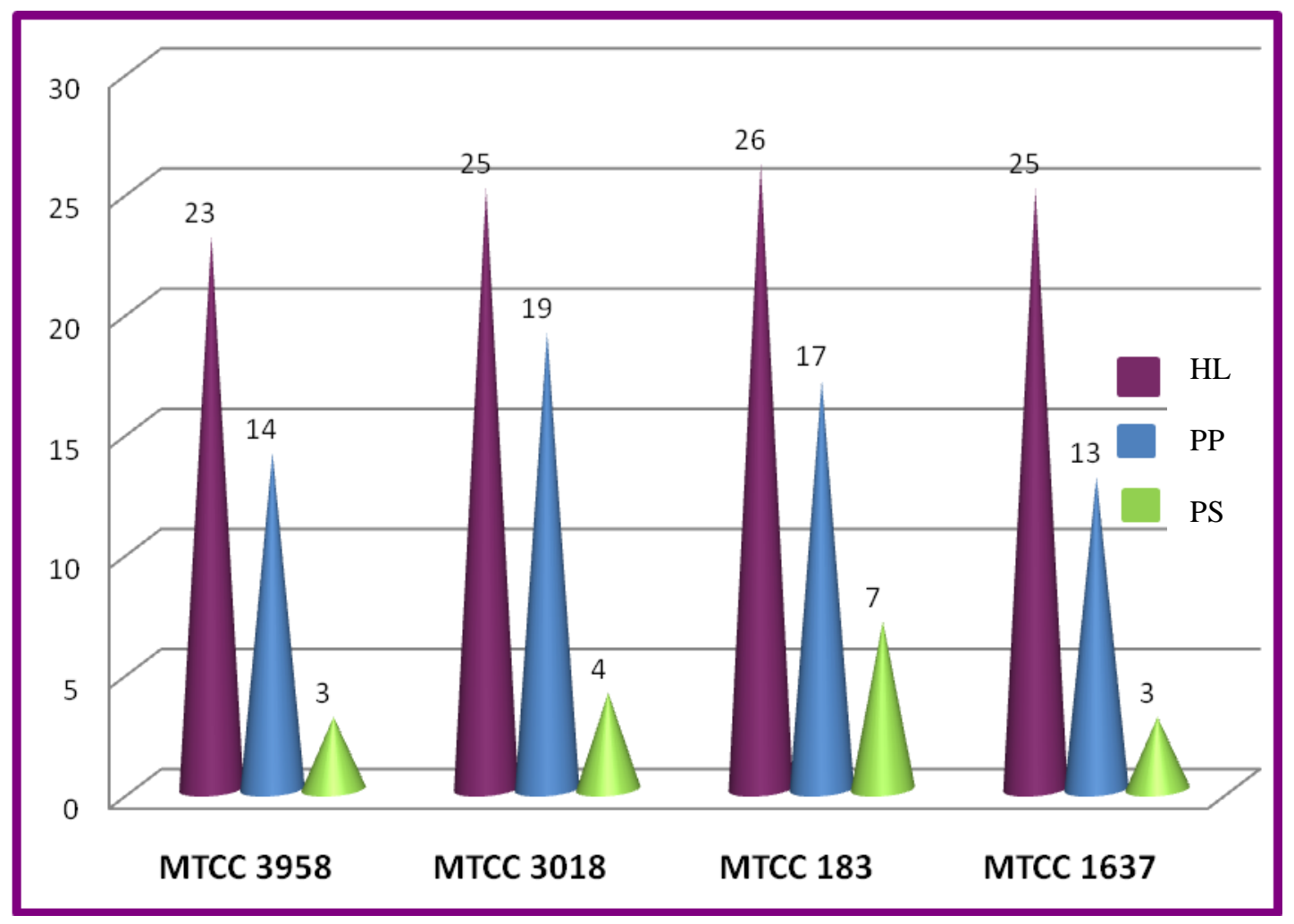

Graph 1: Effect of aqueous extracts of Henna leaves (HL), Pomegranate peels (PP) and Pomegranate seeds (PS) on different strains of Candida Albicans. 


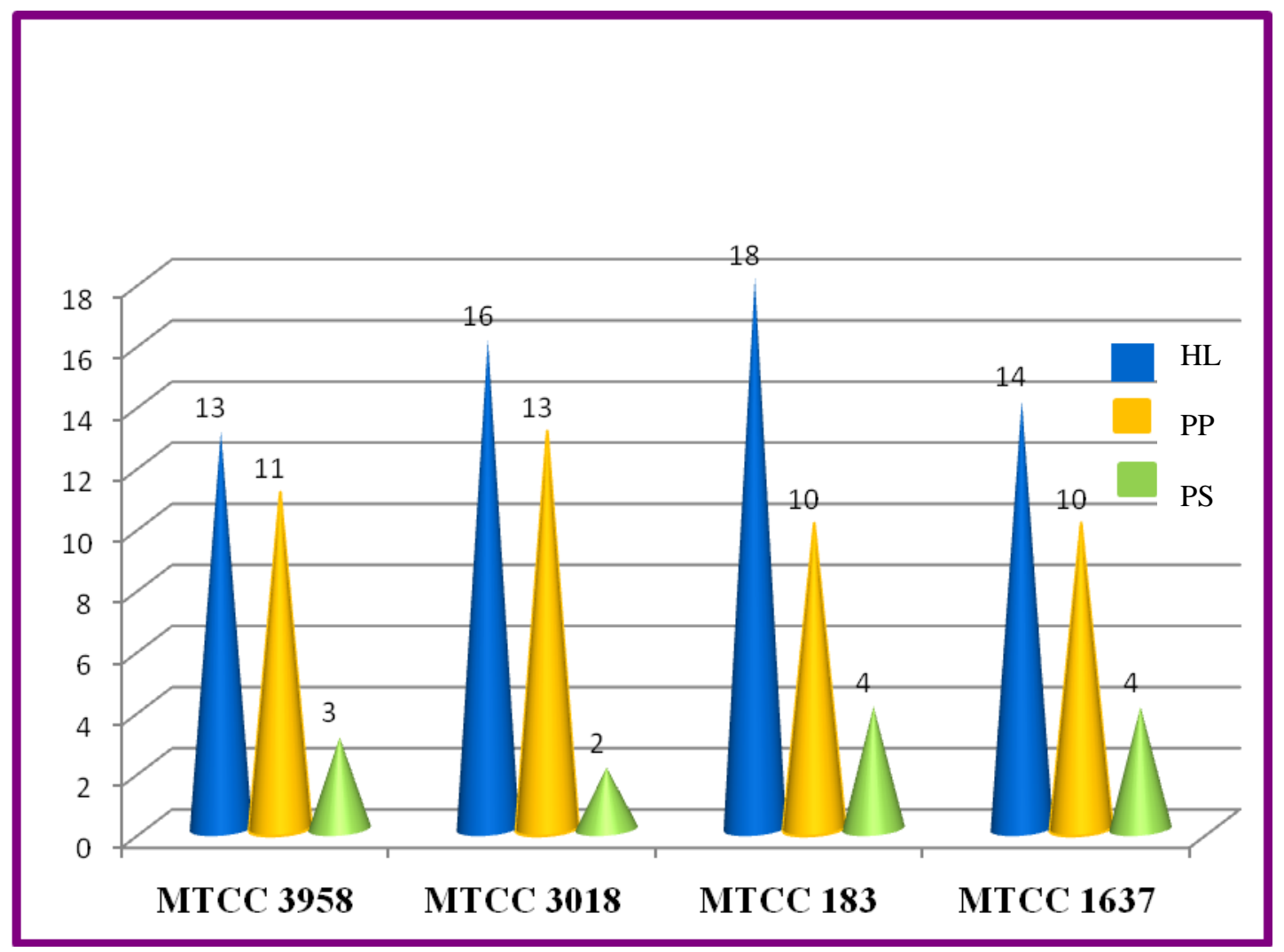

Graph 2: Effect of ethanolic extracts of Henna Leaves (HL), Pomegranate peels (PP) and Pomegranate seeds (PS) on different strains of Candida Albicans.

Recently, fluconazole-resistant C. albicans strains and intrinsically resistant Candida species such as C. glabrata and C. krusei are emerging in immune-compromised patients [7]. Use of natural plant products has opened a new horizon in the treatment of candidiasis. There are three main reasons illustrated in the treating and healing power of plant extract [15].

1) First, pharmacological studies have demonstrated that many of plants are known to possess antimicrobial agents;

2) Second, people are becoming aware of the side effects associated with the over prescription of traditional antibiotics;

3) Third, time to time resistant microorganisms against antibiotic are increasing.

The present study shows the antifungal effect of henna and pomegranate plants. Results of the antifungal screening of different solvent extracts of henna leaves, pomegranate peels and seeds revealed significant antifungal activity against all tested fungal strains.

Henna leaves have showed the maximum antifungal activity against all Candida albicans strains. The effectiveness of henna extracts is shown in table1. Aqueous extracts are more effective on Candida albicans than ethanolic extracts. Fluconazole disc used as standard antifungal drug in a concentration of $20 \mu \mathrm{gm}$ has complete inhibitory effect on Candida albicans. These findings demonstrate that these extracts have antifungal activity in vitro against the fungus which causes oral candidiasis.

Out of the peels and seeds of pomegranate, peels have shown the maximum effect against Candida albicans strains. These results were consistent with the studies conducted by Endo $\mathrm{H}$ who concluded that the fruit peel of Punica granatum was effective for inhibiting Candida albicans growth [16,17].

The results were also in accordance with the report of Vasconcelos et al [18] and Duraipandiyan et al [19] but in contrast with Sh. Abdollahzadeh [15] and Singh's study[20]. Studies conducted by Prasirst have also shown that Henna leaves are effective against oral Candida albicans [21].

Henna contains Lawsone in about 0.5 to $1.5 \%$ of its ingredients. Lawsone (2-hydroxynapthoquinone) is the principal constituent responsible for the dyeing properties of the plant [22]. Lawsone isolated from the leaves of L. inermis has shown significant antifungal antibiotic effect [23]. As the mode of action of lawsone is not clearly understood, ultrastructural studies of Candida exposed to this compound should be performed to determine how the compound affects the organism [24]. 
The antifungal activity of pomegranate peel is attributed to the presence of Punicalgin [25]. Punicalgin is absent in the seeds of pomegranate. Although the exact mechanism of action of Punicalgin (a tannin) is not known but the antifungal effect has been attributed to their toxicity, astringent, molecular structure or other ways [15].

Various Studies conducted in past have demonstrated the antifungal activity individually in pomegranate and henna. But the literature present has not compared the antifungal efficacy of the two agents. While in the present study, apart from demonstrating the antifungal efficacy of the plant extracts, we have made an attempt to analyze and compare the antifungal effects of both henna and pomegranate.

\section{Conclusion}

In view of the present scenario, the use of various plant products as substitutes of antifungal drugs against the diseases caused by Candida albicans have been found to be effective thereby overcoming the side-effects of the known antifungal drugs. Further studies with increased sample size are required to support the herbal approach for management of Candidosis.

\section{Acknowledgements}

I would like to acknowledge Mr. Kanishaq and Miss. Amandeep for their encouraging support and efforts in the completion of this study.

\section{References}

[1] Akpan A, Morgan R. Oral candidiasis. Postgrad Med J 2002;78:455-9.

[2] Epstein JB. Antifungal therapy in oropharyngealmycotic infections. Oral Surg Oral Med Oral Pathol 1990;69:32-41.

[3] Guida RA. Candidiasis of the oropharynx and oesophagus. Ear Nose Throat J 1988;67:832-40.

[4] Ghannoum MA, Radwan SS. Candida adherence to epithelial cells. Boca Raton, FL: CRC Press 1990.

[5] Abu-Elteen KH, Abu-Elteen RM. The prevalence of candida albicans populations in the mouths of complete denture wearers. New Microbiol 1998;21:41-8.

[6] Dangi YS, Soni ML, Namdeo KP. Oral Candidiasis: a review. Int J Pharm Pharm Sci 2010;2(4):36-41.

[7] Casalinuovo IA, DI Francesco P, Garaci E. Fluconazole resistance in Candida albicans: a review of mechanisms. Eur Rev Med Pharmaco 2004;8:69-77.

[8] Abad MJ, Ansuategui M, and Bermejo P. Active antifungal substances from natural sources; ARKIVOC 2007;(vii):116-45.

[9] Amrutesh S. Dentistry \& Ayurveda V - An evidence based approach. IJCDS 2011Feb;2(1):3-9.

[10] Babu PD, Subhasree RS. Antimicrobial Activities of Lawsonia inermis - A Review Acad. J. Plant Sci. 2009;2(4):231-2.

[11] Mastanaiah J, Prabhavathi NB, Varaprasad B. Invitro antibacterial activity of leaf extracts of Lawsonia Inermis. Int. J. Pharm Tech Res. April2011Jun;3(2):1045-9.

[12] Parihar S. Oral Candidiasis- A Review. Webmed Central Dentistry 2011;2(11):1-18.

[13] Millon L, Manteaux A, Reboux G. Fluconazole-resistant recurrent oral candidiasis in human immunodeficiency virus-positive patients: persistence of Candida albicans strains with the same genotype. J Clin Microbiol 1994;32:1115-8.

[14] Rex JH, Rinaldi MG, Pfaller MA. Resistance of Candida species to fluconazole. Antimicrob Agents Chemother 1995;39:1-8.

[15] Sh. Abdollahzade, Mashouf RY, Mortazavi H, Moghaddam MH, Roozbahani N, Vahedi M. Antibacterial and antifungal activities of Punica Granatum peel extracts against oral pathogens. J Dent 2011;8(1):1-6.

[16] Tayel AA, El-Tras WF. Mycoses 2009;53;117-22.

[17] Endo H, Cortez DAG, T. Ueda-Nakamura, Nakamura CV, Filho BPD. Res Microbiol 2010;161:534-40.

[18] Vasconcelos LC, Sampaio MC, Sampaio FC, Higino JS. Use of Punica granatum as an antifungal agent against candidosis associated with denture stomatitis. Mycoses 2003Jun;46(5-6):192-6.

[19] Duraipandiyan V, Ayyanar M, Ignacimuthu S. Antimicrobial activity of some ethnomedicinal plants used by Paliyar tribe from Tamil Nadu, India. BMC Complement Altern Med 2006Oct;6:35.

[20] Singh RP, Chidambara Murthy KN, Jayaprakasha GK. Studies on the antioxidant activity of pomegranate (Punica granatum) peel and seed extracts using in vitro models. J Agric Food Chem 2002Jan;50(1):81-6.

[21] Prasirst J, Leewatthanakorn T, Piamsawad U, Dejrudee A, Panichayupakaranant P, Teanpaisan R, Nittayananta W. Antifungal activity of potassium Lawsone methyl ether mouthwash in comparison with Chlorhexidine mouthwash on oral alternative. Candida isolated from HIV/AIDS subjects. Abstract from 5th World Workshop on Oral Health and Disease in AIDS, Phuket Thailand 2004 Jul:6-9.

[22] Al-Rubiay KK, Jaber NN, Al-Mhaawe BH, Alrubaiy LK. Antimicrobial Efficacy of Henna Extracts. Oman Med J 2008Oct;23(4):253-6.

[23] Chaudhary G, Goyal S, Poonia P. Lawsonia inermis Linnaeus: A Phytopharmacological Review. IJPSDR 2010;2(2):91-8.

[24] Sritrairat N, Nukul N, Inthasame P, Sansuk A, Prasirt J, Leewatthanakorn. Antifungal activity of lawsone methyl ether in comparison with chlorhexidine. J Oral Pathol Med 2011;40:90-6.

[25] Hoda P, Hassanpur S, Emam-djome Z, Lavasani AS. Antimicrobial properties of Pomegranate (Punica granatum L.) as a tannin rich fruit: a review. The 1th International and the 4th National Congress on Recycling of Organic Waste in Agriculture 26 - 27 April 2012 in Isfahan, Iran. 